TRANSACTIONS OF THE

AMERICAN MATHEMATICAL SOCIETY

Volume 348, Number 10, October 1996

\title{
INVARIANTS OF PIECEWISE-LINEAR 3-MANIFOLDS
}

\author{
JOHN W. BARRETT AND BRUCE W. WESTBURY
}

\begin{abstract}
This paper presents an algebraic framework for constructing invariants of closed oriented 3-manifolds by taking a state sum model on a triangulation. This algebraic framework consists of a tensor category with a condition on the duals which we have called a spherical category. A significant feature is that the tensor category is not required to be braided. The main examples are constructed from the categories of representations of involutive Hopf algebras and of quantised enveloping algebras at a root of unity.
\end{abstract}

\section{INTRODUCTION}

The purpose of this paper is to present an algebraic framework for constructing invariants of closed oriented 3-manifolds. The construction is in the spirit of topological quantum field theory and the invariant is calculated from a triangulation of the 3-manifold. The data for the construction of the invariant is a tensor category with a condition on the duals, which we have called a spherical category. The definition of a spherical category and a coherence theorem needed in this paper are given in [3].

There are two classes of examples of spherical categories discussed in this paper. The first examples are given by the quantised enveloping algebra of a semisimple Lie algebra, and the second are given by an involutive Hopf algebra. In the first case, the invariant for $\mathfrak{s l}_{2}$ defined in this paper is the Turaev-Viro invariant [25]. This invariant is known to distinguish lens spaces of the same homotopy type which already shows that the invariants in this paper are not trivial. For the general case, our examples rely on the known properties of the tilting modules, in particular the dimension formula of [1]. The problem of generalising the Turaev-Viro invariant to other quantised enveloping algebras has also been considered by [5], [28] and [24].

A noteworthy feature of our construction is that it does not require a braiding; the notion of a spherical category is more general than the notion of a ribbon category. A simple example of this is the category of representations of the convolution algebra of a non-abelian finite group. This is a spherical category but does not admit a braiding because the representation ring of the algebra is not commutative.

The second class of examples gives a manifold invariant for any involutive Hopf algebra whose dimension is non-zero and finite. It is shown in [4] that this invariant is essentially the same as the invariant defined in [11].

Received by the editors July 20, 1994.

1991 Mathematics Subject Classification. Primary 57N10.

Key words and phrases. 3-manifold invariants, state sum model, bistellar moves, spherical category, spherical Hopf algebra. 
Another feature of this paper is that we prove invariance from a finite list of moves on triangulations. The idea of working directly with triangulations dates from the earlier work of [16] and [14] on the recoupling theory of Lie groups. The moves on triangulations replace the Matveev moves on special spines which are used in [25] and [28]. This approach shows that the invariants are defined for 3-manifolds which may have point singularities of a prescribed type. The two formalisms are however essentially equivalent.

Finally, it is worth noting the cases for which there is a known relationship with the invariants which have been defined using surgery presentations of a framed manifold and invariants of links. It is shown in [27], [22], [20] that the Turaev-Viro invariant is the square of the modulus of the Witten invariant for $\mathfrak{s l}_{2}$ which was defined in [19]. This result has subsequently been generalised by [24] to the case of the invariants defined from a unitary modular category. A modular category is the data required to construct the generalisation of the Reshetikhin-Turaev invariant. While Turaev's work shows that this data determines a state-sum invariant, from our point of view some of this data is redundant. As pointed out above, there are spherical categories which do not admit a braiding. There are also spherical categories which admit a braiding but are not modular, for example the group algebra of a finite group. Direct calculations show that the surgery invariant for the quantum double of the group algebra of a finite group is equal to the invariant defined in this paper for the group algebra itself.

\section{STATe Sum MODELS}

In this paper we have assumed that $\mathbb{F}$ is a field and that a vector space is a finite dimensional vector space over $\mathbb{F}$.

Definition 1.1. A complex is a finite set of elements called vertices, together with a subset of the set of all subsets. These are called simplices. This is required to have the property that any subset of a simplex is a simplex.

Definition 1.2. A simplicial complex is a complex together with a total ordering on the vertices of each simplex such that the ordering on the vertices on any face of a simplex is the ordering induced from the ordering on the vertices of the simplex.

Let $\sigma$ be an $n$-simplex in a simplicial complex. Then for $0 \leqslant i \leqslant n$ define $\partial_{i} \sigma$ to be the face obtained by omitting the $i$-th vertex. These satisfy

$$
\partial_{i} \partial_{j} \sigma=\partial_{j-1} \partial_{i} \sigma \quad \text { if } i<j .
$$

A simplicial complex is an example of the more general notion of simplicial set. This explains the use of the adjective 'simplicial' for the notion of a complex with an ordering. In the following, the adjective 'combinatorial' will be used to refer to complexes, reserving 'simplicial' for simplicial complexes. Combinatorial maps are maps of complexes and simplicial maps are maps of simplicial complexes, i.e., combinatorial maps which preserve orderings. For example, a single simplex considered as a simplicial complex has no symmetries, whereas the corresponding complex admits the permutations as its symmetries.

All manifolds are compact, oriented, piecewise-linear manifolds of dimension three, unless stated otherwise. For background on piecewise-linear manifolds we refer the reader to [21]. In line with the terminology explained above, a simplicial manifold is a simplicial complex whose geometric realisation is a piecewise-linear manifold, together with an orientation. 
Our notation for the orientation of a simplex is fixed as follows. The standard $(n+1)$-simplex $(012 \ldots n)$ with vertices $\{0,1,2, \ldots n\}$ has a standard orientation $(+)$. The opposite orientation is indicated with a minus $(-)$. The standard (oriented) tetrahedron $+(0123)$ has boundary

$$
(123)-(023)+(013)-(012)
$$

The signs indicate the induced orientation of the boundary of $+(0123)$. The tetrahedron - (0123) has the opposite orientation for the boundary,

$$
-(123)+(023)-(013)+(012) \text {. }
$$

In an oriented closed manifold, each triangle is in the boundary of exactly two tetrahedra, with each sign + or - occuring once.

The data for a state sum model consists of three parts, a set of labels $I$, a set of state spaces for a triangle, and a set of partition functions for a tetrahedron.

Definition 1.3. A labelled simplicial complex is a simplicial complex together with a function which assigns an element of $I$ to each edge.

Definition 1.4. Let $T(a, b, c)$ be the standard oriented triangle $+(012)$ labelled by $\partial_{0} T \mapsto a, \partial_{1} T \mapsto b, \partial_{2} T \mapsto c$. The state space for this labelled triangle is a vector space, $H(a, b, c)$. The state space for the oppositely oriented triangle $-T(a, b, c)$ is defined to be the dual vector space, $H^{*}(a, b, c)$.

Definition 1.5. Let $A$ be the standard oriented tetrahedron $+(0123)$ with the edge $\partial_{i} \partial_{j} \sigma$ labelled by $e_{i j}$. The partition function of this labelled tetrahedron is defined to be a linear map

$$
\begin{aligned}
\left\{\begin{array}{lll}
e_{01} & e_{02} & e_{12} \\
e_{23} & e_{13} & e_{03}
\end{array}\right\}_{+}: & \\
& H\left(e_{23}, e_{03}, e_{02}\right) \otimes H\left(e_{12}, e_{02}, e_{01}\right) \rightarrow H\left(e_{23}, e_{13}, e_{12}\right) \otimes H\left(e_{13}, e_{03}, e_{01}\right) .
\end{aligned}
$$

The partition function of $-A$ with the same labelling is defined to be a linear map

$$
\begin{aligned}
\left\{\begin{array}{lll}
e_{01} & e_{02} & e_{12} \\
e_{23} & e_{13} & e_{03}
\end{array}\right\}_{-}: & \\
& H\left(e_{23}, e_{13}, e_{12}\right) \otimes H\left(e_{13}, e_{03}, e_{01}\right) \rightarrow H\left(e_{23}, e_{03}, e_{02}\right) \otimes H\left(e_{12}, e_{02}, e_{01}\right) .
\end{aligned}
$$

In the definition, the four factors in the tensor products correspond to each of the four faces. Also, the two factors in the domain of the linear map correspond to the two faces with sign - in the boundary of the tetrahedron, and the two factors in the range correspond to the two faces with sign + .

Definition 1.6. The data for a state sum model determines an element $Z(M) \in \mathbb{F}$ for each labelled simplicial closed manifold $M$. This is called the simplicial invariant of the labelled manifold. Let $V(M)$ be the tensor product over the set of triangles of $M$ of the state space for each triangle. For each tetrahedron in $M$, take the partition function of the labelled standard tetrahedron, $A$ or $-A$, to which it is isomorphic.

The tensor product over this set of partition functions is a linear map $V(M) \rightarrow$ $V^{\pi}(M)$, where $V^{\pi}(M)$ is defined in the same way as $V(M)$ but with the factors permuted by some permutation $\pi$. This uses the fact that in a closed oriented manifold, each triangle is in the boundary of two tetrahedra, each with opposite 
orientation. There is a unique standard linear map $V^{\pi}(M) \rightarrow V(M)$ given by iterating the standard twist $P: x \otimes y \mapsto y \otimes x$. This defines a linear map $V(M) \rightarrow$ $V(M)$, and the element $Z(M)$ is defined to be the trace of this linear map.

Note that if $A: X \rightarrow Y$ and $B: Y \rightarrow X$ are linear maps, then

$$
\operatorname{tr}^{X \otimes Y}(P(A \otimes B))=\operatorname{tr}^{X}(A B)=\operatorname{tr}^{Y}(B A) .
$$

This also introduces the notation, used throughout the paper, that the map $X$ composed with map $Y$ is written $X Y$ (not $Y \circ X)$.

A state sum invariant of a closed manifold is obtained by a weighted sum of these elements $Z(M)$ over a class of labellings. This state sum invariant is defined in section 5 .

\section{Spherical CATEgories}

The data which defines the state sum model is a spherical category, whose definition is obtained by axiomatising the properties of the category of representations of the spherical Hopf algebra. The reason this abstraction is necessary is that the category of representations of a Hopf algebra may be degenerate, and it is necessary to take a non-degenerate quotient category to construct the invariants.

This quotient is not the category of representations of any finite dimensional Hopf algebra. The reason for this is that it is not possible to assign a positive integer, the dimension, to each object which is additive under direct sum and multiplicative under the tensor product.

First we recall the definition of a strict pivotal category given in [6]. The definition of a (relaxed) pivotal category is given in [3] and a similar definition is given in [7]. A spherical category is a pivotal category which satisfies an additional condition.

In this paper we will only consider strict pivotal categories. There is no loss of generality, as it is shown in [3] that every pivotal category is canonically equivalent to a strict pivotal category. However the main examples of pivotal categories are categories of representations of Hopf algebras and are not strict. The difference between a pivotal category and a strict pivotal category is that some objects that are equal in a strict pivotal category are canonically isomorphic in a pivotal category. In this section we denote any such canonical isomorphism by $=$. These constructions can be extended to pivotal categories by putting in the canonical isomorphism for each $=$.

Definition 2.1. A category with strict duals consists of a category $\mathcal{C}$, a functor $\otimes: \mathcal{C} \times \mathcal{C} \rightarrow \mathcal{C}$, an object $e$ and a functor ${ }^{\wedge}: \mathcal{C} \rightarrow \mathcal{C}^{o p}$. The conditions are that $(\mathcal{C}, \otimes, e)$ is a strict monoidal category and

1. The functors $\widehat{ } \wedge$ and 1 are equal.

2. The objects $\hat{e}$ and $e$ are equal.

3. The functors $\mathcal{C} \times \mathcal{C} \rightarrow \mathcal{C}$ which on objects are given by $(a, b) \mapsto(a \otimes b)^{\wedge}$ and $(a, b) \mapsto \hat{b} \otimes \hat{a}$ are equal.

Definition 2.2. A strict pivotal category is a category with strict duals together with a morphism $\epsilon(c): e \rightarrow c \otimes \hat{c}$ for each object $c \in \mathcal{C}$.

The conditions on the morphisms $\epsilon(c)$ are the following: 
1. For all morphisms, $f: a \rightarrow b$, the following diagram commutes:

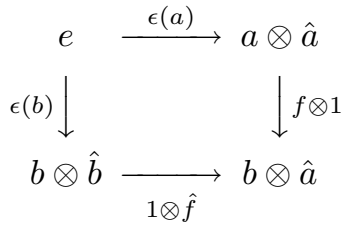

2. For all objects $a$, the following composite is the identity map of $\hat{a}$ :

$$
\hat{a}=e \otimes \hat{a} \stackrel{\epsilon(\hat{a}) \otimes 1}{\longrightarrow}(\hat{a} \otimes \hat{\hat{a}}) \otimes \hat{a}=\hat{a} \otimes(a \otimes \hat{a})^{\curlywedge} \stackrel{1 \otimes \hat{\epsilon}(a)}{\longrightarrow} \hat{a} \otimes \hat{e}=\hat{a} .
$$

3. For all objects $a$ and $b$ the following composite is required to be $\epsilon(a \otimes b)$ :

$$
e \stackrel{\epsilon(a)}{\longrightarrow} a \otimes \hat{a}=a \otimes(e \otimes \hat{a}) \stackrel{1 \otimes(\epsilon(b) \otimes 1)}{\longrightarrow} a \otimes((b \otimes \hat{b}) \otimes \hat{a})=(a \otimes b) \otimes(a \otimes b)^{\wedge} .
$$

The functor ${ }^{\wedge}$ and the maps $\epsilon$ are not independent. The maps $\epsilon$ determine ${ }^{-}$.

Lemma 2.3. In any pivotal category, for any morphism $f: a \rightarrow b$ the following composite is $\hat{f}$ :

$$
\hat{b}=\hat{b} \otimes e \stackrel{1 \otimes \epsilon(a)}{\longrightarrow} \hat{b} \otimes(a \otimes \hat{a}) \stackrel{1 \otimes(f \otimes 1)}{\longrightarrow} \hat{b} \otimes(b \otimes \hat{a})=\widehat{(\hat{b} \otimes \hat{\hat{b}}}) \otimes \hat{a} \stackrel{\hat{\epsilon}(\hat{b}) \otimes 1}{\longrightarrow} \hat{e} \otimes \hat{a}=\hat{a} .
$$

Proof. This follows directly from conditions (1) and (2) of the preceding definition.

Definition 2.4. Let $a$ be any object in a pivotal category. Then the monoid $\operatorname{End}(a)$ has two trace maps, $\operatorname{tr}_{L}, \operatorname{tr}_{R}: \operatorname{End}(a) \rightarrow \operatorname{End}(e)$. In a pivotal category $\operatorname{tr}_{L}(f)$ is defined to be the composite

$$
e \stackrel{\epsilon(\hat{a})}{\longrightarrow} \hat{a} \otimes \hat{\hat{a}}=\hat{a} \otimes a \stackrel{1 \otimes f}{\longrightarrow} \hat{a} \otimes a=(\hat{a} \otimes \hat{\hat{a}})^{-} \stackrel{\hat{\epsilon}(\hat{a})}{\longrightarrow} \hat{e}=e
$$

and $\operatorname{tr}_{R}(f)$ is defined to be the composite

$$
e \stackrel{\epsilon(a)}{\longrightarrow} a \otimes \hat{a} \stackrel{f \otimes 1}{\longrightarrow} a \otimes \hat{a}=(a \otimes \hat{a})^{\wedge} \stackrel{\hat{\epsilon}(a)}{\longrightarrow} \hat{e}=e .
$$

These are called trace maps because they satisfy $\operatorname{tr}_{L}(f g)=\operatorname{tr}_{L}(g f)$ and $\operatorname{tr}_{R}(f g)=$ $\operatorname{tr}_{R}(g f)$.

Definition 2.5. A pivotal category is spherical if, for all objects $a$ and all morphisms $f: a \rightarrow a$,

$$
\operatorname{tr}_{L}(f)=\operatorname{tr}_{R}(f) .
$$

An equivalent condition is that $\operatorname{tr}_{L}(f)=\operatorname{tr}_{L}(\hat{f})$, for all $f: a \rightarrow a$. For each object $a$ in a spherical category, its quantum dimension is defined to be $\operatorname{dim}_{q}(a)=\operatorname{tr}_{L}\left(1_{a}\right)$. Thus, $\operatorname{dim}_{q}(a)=\operatorname{dim}_{q}(\hat{a})$.

Also, in a spherical category, $\operatorname{tr}_{L}(f \otimes g)=\operatorname{tr}_{L}(f) \cdot \operatorname{tr}_{L}(g)$ (where the product is in $\operatorname{End}(e))$ for all $f: a \rightarrow a$ and all $g: b \rightarrow b$.

All spherical categories considered in the rest of this are additive. This means that each Hom set is a finitely generated abelian group with composition $\mathbb{Z}$-bilinear and that the data defining the spherical structure is compatible with the additive structure. This means that $\otimes$ is $\mathbb{Z}$-bilinear and that ${ }^{\wedge}$ is $\mathbb{Z}$-linear.

In any additive monoidal category $\operatorname{End}(e)$ is a commutative ring (see [10]) and we denote this ring by $\mathbb{F}$. In particular each of the above trace maps takes values in this ring. It follows that an additive monoidal category is $\mathbb{F}$-linear. We need to assume 
some further conditions. These are that $\mathbb{F}$ is a field and that each set of morphisms is a finite dimensional vector space over $\mathbb{F}$. In this paper an additive spherical category means a spherical category in which these conditions are satisfied.

The main examples of additive spherical categories arise as the category of representations of a Hopf algebra with some additional structure. This is discussed in section 6 .

Example 2.6. An example of a spherical category which cannot be regarded as a category whose objects are finite dimensional vector spaces is given by taking the free $\mathbb{Z}[\delta, z]$-linear category on the category of oriented framed tangles and then taking the quotient by the well-known skein relation for the HOMFLY polynomial. This is a spherical category, and for each pair of objects $X$ and $Y, \operatorname{Hom}(X, Y)$ is a finitely generated free module. This example satisfies all the conditions for an additive spherical category except that $\mathbb{F}$ is not a field. However the objects cannot be taken to be finitely generated modules unless $z$ is a quantum integer.

Strict pivotal categories are discussed in [6], where it is shown that the category of oriented planar graphs up to isotopy and with labelled edges is a strict pivotal category. Similar constructions are discussed [9], [7], [19]. The following is an informal statement of the result needed in the discussion in this paper. This result is not required for the proofs in this paper, as an algebraic proof can always be given in place of a diagrammatic proof. However it is important for an understanding of the proofs.

Given a trivalent planar graph with the following data attached:

1. An orientation of each edge,

2. A distinguished edge at each trivalent vertex,

3. A map from edges to objects,

4. A map from vertices to morphisms,

then this graph can be evaluated to give a morphism. The relations for a pivotal category imply that this evaluation depends only on the isotopy class of the graph, where the attached data is carried along with the isotopy.

The sphere $S^{2}$ can be regarded as the plane with the point at infinity attached, and so a planar graph can also be thought of as a graph embedded in $S^{2}$. Spherical categories are pivotal categories which satisfy an extra condition, the equality of left and right trace. This condition implies that the spherical category determines an invariant of isotopy classes of closed graphs on the sphere. There is an isotopy of the sphere which takes a closed graph of the form of Figure 1 to the graph obtained by closing $M$ in a loop to the left. This isotopy moves the loop in Figure 1 past the point at infinity. Taken together with planar isotopies, such an operation on planar graphs generates all the isotopies on the sphere.

Definition 2.7. For any two objects $a$ and $b$ there is a bilinear pairing

$$
\Theta: \operatorname{Hom}(a, b) \times \operatorname{Hom}(b, a) \rightarrow \mathbb{F}
$$

defined by $\Theta(f, g)=\operatorname{tr}_{L}(f g)=\operatorname{tr}_{L}(g f)$.

Definition 2.8. An additive spherical category is non-degenerate if, for all objects $a$ and $b$, the pairing $\Theta$ is non-degenerate.

The next theorem shows that every additive spherical category has a natural quotient which is a non-degenerate spherical category. 


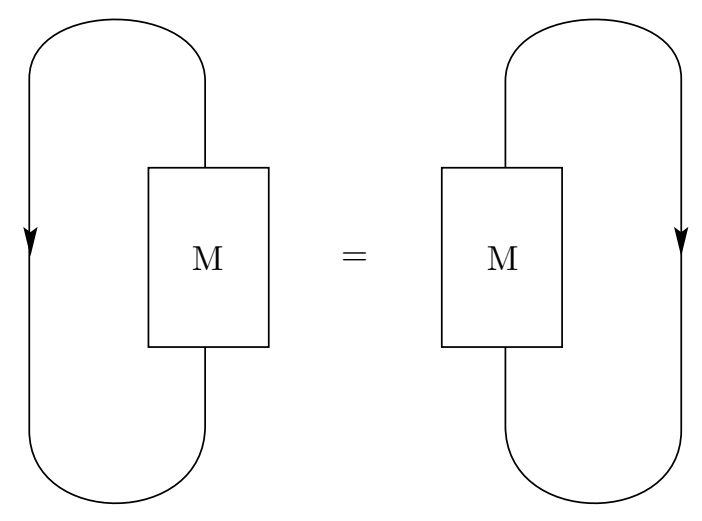

Figure 1

Theorem 2.9. Let $\mathcal{C}$ be an additive spherical category. Define the additive subcategory $\mathcal{J}$ to have the same set of objects and morphisms defined by

$$
\operatorname{Hom}_{\mathcal{J}}\left(c_{1}, c_{2}\right)=\left\{f \in \operatorname{Hom}_{\mathcal{C}}\left(c_{1}, c_{2}\right): \operatorname{tr}_{L}(f g)=0 \text { for all } g \in \operatorname{Hom}_{\mathcal{C}}\left(c_{2}, c_{1}\right)\right\} \text {. }
$$

Then $\mathcal{C} / \mathcal{J}$ is a non-degenerate additive spherical category.

Proof. It is clear that $\mathcal{J}$ is closed under composition on either side by arbitrary morphisms in $\mathcal{C}$. Hence the quotient is an additive category. It is also clear that $\hat{f} \in \mathcal{J}$ if and only if $f \in \mathcal{J}$, and so the functor ${ }^{\wedge}$ is well-defined on the quotient. The functor $\otimes$ is well-defined on the quotient, since $f \in \mathcal{J}$ implies $f \otimes g_{1} \in \mathcal{J}$ and $g_{2} \otimes f \in \mathcal{J}$ for arbitrary morphisms in $\mathcal{C}$. This follows from the observation that $\operatorname{tr}_{L}(f \otimes g)=\operatorname{tr}_{L}(f) \cdot \operatorname{tr}_{L}(g)$, which uses the spherical condition.

The morphisms $\epsilon(a)$ are taken to be the images in the quotient of the given morphisms in $\mathcal{C}$. The conditions on this structure which imply that this quotient is spherical follow from the same conditions in $\mathcal{C}$.

Each pairing $\Theta$ is non-degenerate by construction.

An extra condition on the spherical category is required for the piecewise-linear invariance of the partition function of a natural simplicial field theory. Similar conditions have been considered by [17], [27], [26], [23] and [28]. An object $a$ is called non-zero if the ring $\operatorname{End}(a) \neq 0$.

Definition 2.10. A semisimple spherical category is an additive, non-degenerate, spherical category such that there exists a set of inequivalent non-zero objects, $J$, such that for any two objects $x$ and $y$, the natural map given by composition,

$$
\bigoplus_{a \in J} \operatorname{Hom}(x, a) \otimes \operatorname{Hom}(a, y) \rightarrow \operatorname{Hom}(x, y),
$$

is an isomorphism.

An object $a$ is called simple if $\operatorname{End}(a) \cong \mathbb{F}$.

The following lemma shows that the set $J$ is essentially fixed by the category.

Lemma 2.11. Every simple object is isomorphic to a unique element of $J$, and every element of $J$ is simple. 
Proof. In the formula

$$
\bigoplus_{a \in J} \operatorname{Hom}(x, a) \otimes \operatorname{Hom}(a, x) \cong \operatorname{End}(x)
$$

first consider $x$ to be an element of $J$. Then by counting dimensions, one has that $\operatorname{End}(x) \cong \mathbb{F}$.

Now consider the same formula with $x$ any simple object. Again by counting dimensions, only one of the terms on the left is non-zero. For this $a \in J, \operatorname{Hom}(x, a) \cong$ $\operatorname{Hom}(a, x) \cong \mathbb{F}$. Thus there are elements $f \in \operatorname{Hom}(x, a), g \in \operatorname{Hom}(a, x)$ such that $f g=i d_{x}$. From this it follows that $g f \in \operatorname{End}(a)$ is an idempotent and is not zero. But $\operatorname{End}(a) \cong \mathbb{F}$, and so $g f=i d_{a}$. This shows that $x$ is isomorphic to $a \in J$.

Definition 2.12. A semisimple spherical category is called finite if the set of isomorphism classes of simple objects is finite.

Definition 2.13. The dimension $K$ of a finite semisimple spherical category is defined by the formula

$$
K=\sum_{a \in J} \operatorname{dim}_{q}^{2}(a)
$$

for some choice $J$ of one object in each isomorphism class of simple objects. The dimension is independent of this choice.

Lemma 2.14. For each pair of objects $(a, b)$ in a semisimple spherical category,

$$
\operatorname{dim}_{q}(a) \operatorname{dim}_{q}(b)=\sum_{c \in J} \operatorname{dim}_{q}(c) \operatorname{dim} \operatorname{Hom}(c, a \otimes b) .
$$

Proof. The left-hand side is equal to tr $1_{a \otimes b}$. The lemma follows from the application of the semisimple condition of Definition 2.10 with $x=y=a \otimes b$, and some linear algebra.

\section{Symmetries of Simplicial InVARIANTS}

In this section, we define the data for a state sum model given a strict nondegenerate spherical category $\mathcal{C}$. Then we show that the simplicial invariant of labelled manifolds has the property that it depends only on the isomorphism class of the labelling of each edge. Then it is shown that the invariant depends only on the underlying combinatorial structure of the simplicial complex.

The data for a state sum model is constructed as follows. The label set $I$ is the set of simple objects in the category. For each ordered triple $(a, b, c)$ of labels, the vector space $H(a, b, c)$ is defined to be $\operatorname{Hom}(b, a \otimes c)$ (see Figure 2).

For the partition function of the tetrahedron $A$ labelled by $e_{01}=a, e_{02}=b$, $e_{12}=c, e_{23}=d, e_{13}=e, e_{03}=f$, first define a linear functional on the space

$$
\operatorname{Hom}(d \otimes c, e) \otimes \operatorname{Hom}(f, d \otimes b) \otimes \operatorname{Hom}(e \otimes a, f) \otimes \operatorname{Hom}(b, c \otimes a) .
$$

The linear functional is defined to be

$$
\alpha \otimes \beta \otimes \gamma \otimes \delta \mapsto \operatorname{tr}_{L}(\beta(1 \otimes \delta)(\alpha \otimes 1) \gamma)
$$

(Figure 3). This linear functional determines a unique linear map

$$
\left\{\begin{array}{lll}
a & b & c \\
d & e & f
\end{array}\right\}_{+}: \operatorname{Hom}(f, d \otimes b) \otimes \operatorname{Hom}(b, c \otimes a) \rightarrow \operatorname{Hom}(e, d \otimes c) \otimes \operatorname{Hom}(f, e \otimes a)
$$




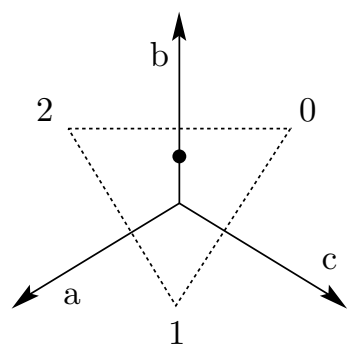

Figure 2

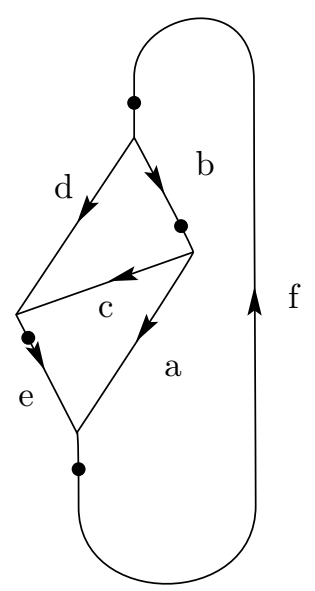

FigURE 3

using the non-degenerate pairings

$$
\operatorname{Hom}^{*}(d \otimes c, e) \cong \operatorname{Hom}(e, d \otimes c) \text { and } \operatorname{Hom}^{*}(e \otimes a, f) \cong \operatorname{Hom}(f, e \otimes a) .
$$

For the partition function of $-A$ labelled in the same way, the linear functional on

$$
\operatorname{Hom}(e, d \otimes c) \otimes \operatorname{Hom}(d \otimes b, f) \otimes \operatorname{Hom}(f, e \otimes a) \otimes \operatorname{Hom}(c \otimes a, b)
$$

defined by

$$
\alpha \otimes \beta \otimes \gamma \otimes \delta \mapsto \operatorname{tr}_{L}(\gamma(\alpha \otimes 1)(1 \otimes \delta) \beta) .
$$

likewise determines a unique linear map

$$
\left\{\begin{array}{lll}
a & b & c \\
d & e & f
\end{array}\right\}_{-}: \operatorname{Hom}(e, d \otimes c) \otimes \operatorname{Hom}(f, e \otimes a) \rightarrow \operatorname{Hom}(f, d \otimes b) \otimes \operatorname{Hom}(b, c \otimes a)
$$

Definition 3.1. Given isomorphisms $\phi_{a}: a \rightarrow a^{\prime}, \phi_{b}: b \rightarrow b^{\prime}$ and $\phi_{c}: c \rightarrow c^{\prime}$, there is an induced isomorphism

$$
\operatorname{Hom}(b, a \otimes c) \rightarrow \operatorname{Hom}\left(b^{\prime}, a^{\prime} \otimes c^{\prime}\right)
$$

given by $\alpha \mapsto \phi_{b}^{-1} \alpha\left(\phi_{a} \otimes \phi_{c}\right)$. 
Lemma 3.2. Given any ordered 6-tuple of elements of $I,(a, b, c, d, e, f)$, and an ordered 6-tuple of isomorphisms, $\left(\phi_{a}, \phi_{b}, \phi_{c}, \phi_{d}, \phi_{e}, \phi_{f}\right)$, where $\phi_{a}: a \rightarrow a^{\prime}, \ldots, \phi_{f}: f$ $\rightarrow f^{\prime}$, then the following diagram commutes:

$$
\begin{aligned}
& \operatorname{Hom}(f, d \otimes b) \otimes \operatorname{Hom}(b, c \otimes a) \longrightarrow \operatorname{Hom}\left(f^{\prime}, d^{\prime} \otimes b^{\prime}\right) \otimes \operatorname{Hom}\left(b^{\prime}, c^{\prime} \otimes a^{\prime}\right) \\
& \left\{\begin{array}{lll}
a & b & c \\
d & e & f
\end{array}\right\} \downarrow \downarrow \downarrow\left\{\begin{array}{lll}
a^{\prime} & b^{\prime} & c^{\prime} \\
d^{\prime} & e^{\prime} & f^{\prime}
\end{array}\right\} \\
& \operatorname{Hom}(e, d \otimes c) \otimes \operatorname{Hom}(f, e \otimes a) \longrightarrow \operatorname{Hom}\left(e^{\prime}, d^{\prime} \otimes c^{\prime}\right) \otimes \operatorname{Hom}\left(f^{\prime}, e^{\prime} \otimes a^{\prime}\right)
\end{aligned}
$$

Also, the diagram for the opposite orientation commutes:

$$
\begin{aligned}
& \operatorname{Hom}(f, d \otimes b) \otimes \operatorname{Hom}(b, c \otimes a) \longrightarrow \operatorname{Hom}\left(f^{\prime}, d^{\prime} \otimes b^{\prime}\right) \otimes \operatorname{Hom}\left(b^{\prime}, c^{\prime} \otimes a^{\prime}\right) \\
& \left\{\begin{array}{lll}
a & b & c \\
d & e & f
\end{array}\right\} \uparrow \uparrow \uparrow\left\{\begin{array}{lll}
a^{\prime} & b^{\prime} & c^{\prime} \\
d^{\prime} & e^{\prime} & f^{\prime}
\end{array}\right\} \\
& \operatorname{Hom}(e, d \otimes c) \otimes \operatorname{Hom}(f, e \otimes a) \longrightarrow \operatorname{Hom}\left(e^{\prime}, d^{\prime} \otimes c^{\prime}\right) \otimes \operatorname{Hom}\left(f^{\prime}, e^{\prime} \otimes a^{\prime}\right)
\end{aligned}
$$

Proof. First, in the diagram

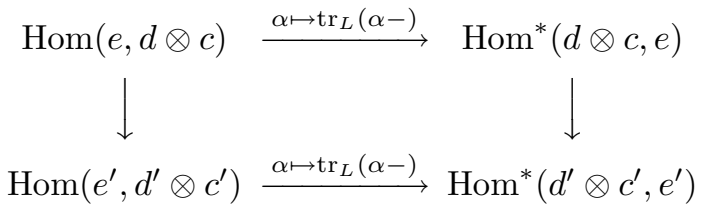

the horizontal arrows are defined by the pairings, the left vertical arrow by the induced isomorphism of the previous definition, and the right vertical arrow by the adjoint of the map $\beta \mapsto\left(\phi_{e} \otimes \phi_{a}\right) \beta \phi_{f}^{-1}$. This diagram, and a similar diagram obtained by replacing $e, d, c$ with $f, e, a$, commute. These diagrams are used to compute the action of the isomorphisms of the statement of the lemma on the linear functionals in the definition of the partition function.

The first diagram in the statement of the lemma commutes as a consequence of the identity

$$
\begin{array}{r}
\operatorname{tr}_{L} \phi_{f}^{-1} \beta\left(\phi_{d} \otimes \phi_{b}\right)\left(1 \otimes\left(\phi_{b}^{-1} \delta\left(\phi_{c} \otimes \phi_{a}\right)\right)\right)\left(\left(\left(\phi_{d} \otimes \phi_{c}\right)^{-1} \alpha \phi_{e}\right) \otimes 1\right)\left(\phi_{e} \otimes \phi_{a}\right)^{-1} \gamma \phi_{f} \\
=\operatorname{tr}_{L}(\beta(1 \otimes \delta)(\alpha \otimes 1) \gamma) .
\end{array}
$$

The proof that the second diagram commutes is similar.

Proposition 3.3. Let $M$ be a closed simplicial manifold. Let $l_{1}$ and $l_{2}$ be two labellings such that the two labels associated to any edge are isomorphic. Then, $Z\left(M, l_{1}\right)=Z\left(M, l_{2}\right)$.

Proof. According to the previous lemma, the map $V(M) \rightarrow V(M)$ is conjugated by the induced isomorphism on the state space of each triangle. The invariant $Z(M)$ is the trace of this map and is invariant under conjugation by a linear map.

Next, we determine the behaviour of the simplicial invariant $Z(M)$ under combinatorial maps. For this, it is necessary to use the properties of duals in the spherical category.

Definition 3.4. Let $f: M \rightarrow N$ be a combinatorial isomorphism of simplicial complexes. Let $e$ be any edge of $M$, labelled by $a$, and let $b$ be the label of edge $f(e)$ in $N$. Then $f$ is compatible with these labellings if $b=a$ in the case that $f$ preserves the orientation of the edge, and $b=\hat{a}$ in the case that $f$ reverses the orientation. 


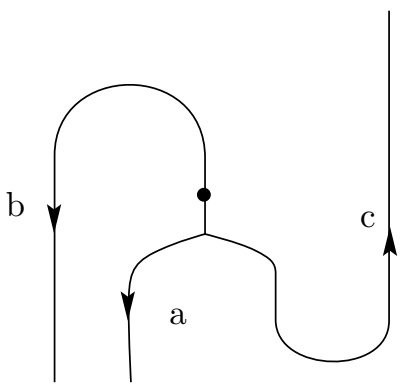

Figure 4

Note that, given $f$ and a labelling of $M$, there is a unique compatible labelling of $N$.

Now the properties of the state space of a triangle under combinatorial isomorphisms are described. The combinatorial isomorphisms are just permutations in $S_{3}$. For the standard triangle $T(a, \hat{b}, c)$, labelled by $\partial_{0} T \mapsto a, \partial_{1} T \mapsto \hat{b}, \partial_{2} T \mapsto c$, the labelling is permuted by $(a, b, c) \mapsto \sigma^{+}(a, b, c)$ for an even permutation $\sigma^{+}$, and $(a, b, c) \mapsto \sigma^{-}(\hat{a}, \hat{b}, \hat{c})$ for an odd permutation $\sigma^{-}$. For this reason, it is more convenient to use the notation $V(a, b, c)=H(a, \hat{b}, c)$ for the state space of a labelled triangle when the symmetry properties are considered.

There is a canonical map $V(a, b, c) \rightarrow V(b, c, a)$, i.e.,

$$
\operatorname{Hom}(\hat{b}, a \otimes c) \rightarrow \operatorname{Hom}(\hat{c}, b \otimes a),
$$

defined by mapping $f: \hat{b} \rightarrow a \otimes c$ to the following composite:

$$
\hat{c} \stackrel{\equiv}{\longrightarrow} e \hat{c} \stackrel{\epsilon(b) \otimes 1}{\longrightarrow} b \otimes \hat{b} \otimes \hat{c} \stackrel{1 \otimes f \otimes 1}{\longrightarrow} b \otimes a \otimes c \otimes \hat{c} \stackrel{1 \otimes 1 \otimes \hat{\epsilon}(c)}{\longrightarrow} b \otimes a \otimes e \stackrel{\equiv}{\longrightarrow} b \otimes a .
$$

This corresponds to the graph in Figure 4.

There is also a canonical pairing $V(a, b, c) \times V(\hat{c}, \hat{b}, \hat{a}) \rightarrow \mathbb{F}$, i.e.,

$$
\operatorname{Hom}(\hat{b}, a \otimes c) \times \operatorname{Hom}(b, \hat{c} \otimes \hat{a}) \rightarrow \mathbb{F} .
$$

Let $f: \hat{b} \rightarrow a \otimes c$ and $g: b \rightarrow \hat{c} \otimes \hat{a}$. Then the pairing is defined by

$$
\langle f, g\rangle=\operatorname{tr}_{L}(f \hat{g}) \text {. }
$$

Equivalently, it is determined by the closed tangle in Figure 5.

Definition 3.5. For every ordered triple, $(a, b, c)$, of elements of $I$ and every even permutation $\sigma^{+} \in S_{3}$, there is an isomorphism

$$
\theta\left(\sigma^{+}\right): V(a, b, c) \rightarrow V \sigma^{+}(a, b, c) .
$$

For $(a, b, c) \mapsto(b, c, a)$, this is the canonical map just defined. Repeating this gives the isomorphism for $(a, b, c) \mapsto(c, a, b)$. The identity is associated to the identity.

For every ordered triple, $(a, b, c)$, of elements of $I$, and every odd permutation $\sigma^{-} \in S_{3}$, there is a non-degenerate pairing, $\langle-,-\rangle_{\sigma^{-}}$,

$$
V(a, b, c) \otimes V \sigma^{-}(\hat{a}, \hat{b}, \hat{c}) \rightarrow \mathbb{F} .
$$

For $\sigma^{-}$the odd permutation $(a, b, c) \mapsto(c, b, a)$, this is the pairing defined above. The pairings for the other two odd permutations can be defined by the formula $\left\langle v_{1}, v_{2}\right\rangle_{\sigma^{-}}=\left\langle v_{1}, \theta\left(\sigma^{+}\right) v_{2}\right\rangle_{\sigma^{-} \sigma^{+}}$. 


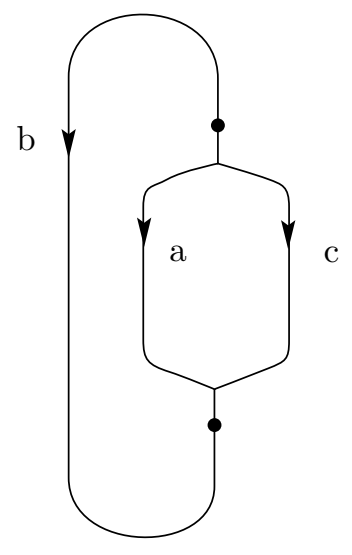

Figure 5

Lemma 3.6. For all even permutations $\sigma_{1}^{+}, \sigma_{2}^{+}$, odd permutations $\sigma^{-}$, labels $a, b$ and $c$ and all $v_{1} \in V(a, b, c)$ and $v_{2} \in V \sigma^{-}(\hat{a}, \hat{b}, \hat{c})$ :

$$
\begin{aligned}
\theta\left(\sigma_{1}^{+} \sigma_{2}^{+}\right) & =\theta\left(\sigma_{1}^{+}\right) \theta\left(\sigma_{2}^{+}\right), \\
\left\langle v_{1}, v_{2}\right\rangle_{\sigma^{-}} & =\left\langle v_{1}, \theta\left(\sigma^{+}\right) v_{2}\right\rangle_{\sigma^{-} \sigma^{+}}, \\
\left\langle v_{1}, v_{2}\right\rangle_{\sigma^{-}} & =\left\langle v_{2}, v_{1}\right\rangle_{\sigma^{-}} .
\end{aligned}
$$

Also, the pairings are non-degenerate bilinear forms.

Proof. The first two relations follow from the fact that the following composite is the identity map:

$$
V(a, b, c) \rightarrow V(b, c, a) \rightarrow V(c, a, b) \rightarrow V(a, b, c) .
$$

This condition is the relation shown in Figure 6, which is satisfied in any pivotal category, by Lemma 2.3 .

The pairings are non-degenerate since the spherical category is non-degenerate and $^{\wedge}$ is an isomorphism on spaces of morphisms. The pairing $\operatorname{tr}_{L}(f \hat{g})$ is symmetric since

$$
\operatorname{tr}_{L}(g \hat{f})=\operatorname{tr}_{L}(\widehat{g \hat{f}})=\operatorname{tr}_{L}(f \hat{g}) .
$$

The symmetry of the other pairings is equivalent to the relations

$$
\operatorname{tr}_{L}\left(\left(\theta\left(\sigma^{+}\right) v_{2}\right) \hat{v}_{1}\right)=\operatorname{tr}_{L}\left(\left(\theta\left(\sigma^{+}\right) v_{1}\right) \hat{v}_{2}\right) .
$$

This follows from the relations in a spherical category, as can be seen by the isotopy equivalence of the corresponding diagrams.

The union of the spaces $\left\{V(a, b, c) \amalg V^{*}(a, b, c) \mid(a, b, c) \in I \times I \times I\right\}$ forms a vector bundle over $I \times I \times I \times\{ \pm\}$. The permutation group, $S_{3}$, acts on the base space by

$$
\begin{aligned}
\sigma^{+}:(a, b, c, \pm) & \mapsto\left(\sigma^{+}(a, b, c), \pm\right), \\
\sigma^{-}:(a, b, c, \pm) & \mapsto\left(\sigma^{-}(\hat{a}, \hat{b}, \hat{c}), \mp\right) .
\end{aligned}
$$




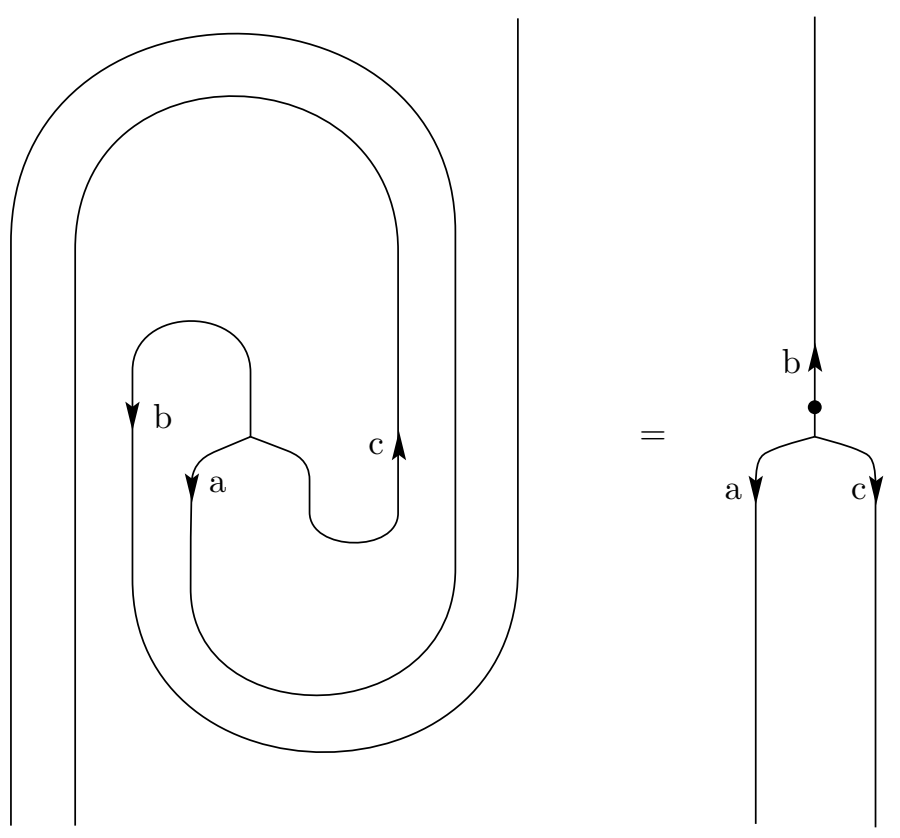

Figure 6

Definition 3.7. For each triple of labels, $(a, b, c)$, and each permutation $\sigma^{+}$or $\sigma^{-}$ there are linear isomorphisms

$$
\begin{gathered}
V(a, b, c) \stackrel{\theta\left(\sigma^{+}\right)}{\longrightarrow} V \sigma^{+}(a, b, c), \\
V^{*}(a, b, c) \stackrel{\theta^{*-1}\left(\sigma^{+}\right)}{\longrightarrow} V^{*} \sigma^{+}(a, b, c),
\end{gathered}
$$

if $\sigma^{+}$is even, and

$$
\begin{gathered}
V(a, b, c) \stackrel{\theta\left(\sigma^{-}\right)}{\longrightarrow} V^{*} \sigma^{-}(\hat{a}, \hat{b}, \hat{c}), \\
V^{*}(a, b, c) \stackrel{\theta^{*-1}\left(\sigma^{-}\right)}{\longrightarrow} V \sigma^{-}(\hat{a}, \hat{b}, \hat{c}),
\end{gathered}
$$

if $\sigma^{-}$is odd. The maps $\theta\left(\sigma^{-}\right)$are defined using the pairings.

Lemma 3.8. These linear maps determine an action of the group $S_{3}$ on this vector bundle, or, in other words, this is an $S_{3}$-equivariant vector bundle. Furthermore, the action of any permutation on elements of $V^{*}(a, b, c)$ is the adjoint of the inverse of the action on $V(a, b, c)$.

Proof. These are equivalent to the conditions in Lemma 3.6.

Theorem 3.9. Let $f: M \rightarrow N$ be a combinatorial isomorphism of labelled manifolds. Then the simplicial invariants are equal, $Z(M)=Z(N)$.

Proof. Let $V(M)$ and $V(N)$ be the vector spaces described in Definition 1.6. For each triangle in $M$, consider the restriction of $f$ to this triangle. There is a element $\sigma \in S_{3}$ defined by the unique decomposition of this map into a permutation followed by the simplicial map of the triangle to its image in $N$. 
There is a map

$$
V(M) \otimes V^{*}(M) \rightarrow V(N) \otimes V^{*}(N)
$$

which is defined by taking the tensor product over the set of triangles of the maps

$$
\theta(\sigma) \otimes \theta^{*-1}(\sigma)
$$

for each triangle, followed by an iteration of the standard twist $P$ which rearranges the factors in the range of this map to coincide with $V(N) \otimes V^{*}(N)$, as in Definition 1.6. Since the action of $\sigma$ on $V^{*}\left(e_{1}, e_{2}, e_{3}\right)$ is the inverse of the adjoint of the action on $V\left(e_{1}, e_{2}, e_{3}\right)$, it follows that the diagram

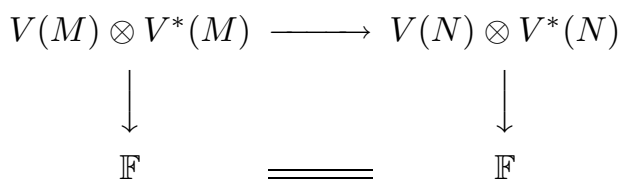

in which the vertical maps are the canonical pairings, commutes.

To complete the proof of the theorem, it remains to show that the map $V(M) \rightarrow$ $V(M)$ whose trace is $Z(M)$ is preserved under this mapping. That is, that this element of $V(M) \otimes V^{*}(M)$ is mapped to the corresponding element of $V(N) \otimes$ $V^{*}(N)$. According to Definition 1.6, each of these elements is the tensor product of partition functions for each tetrahedron. Thus it is sufficient to show that the partition function of the standard tetrahedron is preserved under a combinatorial mapping. This is demonstrated by the next lemma.

Definition 3.10. Let $T$ be an oriented labelled simplicial surface. Then the state space of $T$ is defined to be the tensor product over the set of triangles of the state space for each oriented triangle.

Let $T \rightarrow U$ be an orientation-preserving combinatorial isomorphism of oriented labelled simplicial surfaces which is compatible with the labellings. Then there is a linear isomorphism from the state space of $T$ to the state space of $U$. On each triangle in $T$ an element of $S_{3}$ is determined such that the combinatorial map is a permutation followed by a simplicial map. The linear isomorphism is defined by taking the tensor product over triangles of the linear isomorphisms of Definition 3.7. This tensor product is composed with the unique iterate of the twist map $P$ which has its range the state space of $U$.

Lemma 3.11. The partition function of the standard labelled tetrahedra $A$ and $-A$ are elements of the state spaces of their boundary. Let $\Sigma \in S_{4}$ be an even permutation. The element $\Sigma$ determines combinatorial maps $A \rightarrow A^{\prime}$ and $-A \rightarrow$ $-A^{\prime}$, where $A^{\prime}$ is labelled by a compatible labelling $\left\{e_{i j}^{\prime}\right\}$. Under the linear map of state spaces,

$$
\left\{\begin{array}{lll}
e_{01} & e_{02} & e_{12} \\
e_{23} & e_{13} & e_{03}
\end{array}\right\}_{+} \mapsto\left\{\begin{array}{lll}
e_{01}^{\prime} & e_{02}^{\prime} & e_{12}^{\prime} \\
e_{23}^{\prime} & e_{13}^{\prime} & e_{03}^{\prime}
\end{array}\right\}_{+}
$$

and

$$
\left\{\begin{array}{lll}
e_{01} & e_{02} & e_{12} \\
e_{23} & e_{13} & e_{03}
\end{array}\right\}_{-} \mapsto\left\{\begin{array}{lll}
e_{01}^{\prime} & e_{02}^{\prime} & e_{12}^{\prime} \\
e_{23}^{\prime} & e_{13}^{\prime} & e_{03}^{\prime}
\end{array}\right\}_{-}
$$


If $\Sigma$ is an odd permutation, then

$$
\left\{\begin{array}{lll}
e_{01} & e_{02} & e_{12} \\
e_{23} & e_{13} & e_{03}
\end{array}\right\}_{+} \mapsto\left\{\begin{array}{lll}
e_{01}^{\prime} & e_{02}^{\prime} & e_{12}^{\prime} \\
e_{23}^{\prime} & e_{13}^{\prime} & e_{03}^{\prime}
\end{array}\right\}
$$

and

$$
\left\{\begin{array}{lll}
e_{01} & e_{02} & e_{12} \\
e_{23} & e_{13} & e_{03}
\end{array}\right\}_{-} \mapsto\left\{\begin{array}{lll}
e_{01}^{\prime} & e_{02}^{\prime} & e_{12}^{\prime} \\
e_{23}^{\prime} & e_{13}^{\prime} & e_{03}^{\prime}
\end{array}\right\}_{+}
$$

Proof. The first statement follows from the isomorphism $\operatorname{Hom}(X, Y) \cong X^{*} \otimes Y$ for vector spaces $X, Y$.

In the definition of the partition function of the tetrahedron, each factor $\operatorname{Hom}(\hat{b}, a \otimes c)$ in the tensor product is identified with $\operatorname{Hom}^{*}(a \otimes c, \hat{b})$, using the nondegenerate symmetric pairing $(\alpha, \beta) \mapsto \operatorname{tr}_{L}(\alpha \beta)$ in the spherical category. Using these isomorphisms, the action of the odd and even permutations can be computed by the following commutative diagrams:

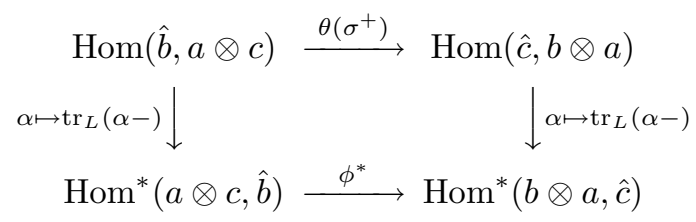

in which $\phi: \beta \mapsto \widehat{\theta\left(\sigma^{+}\right)} \hat{\beta}$, and

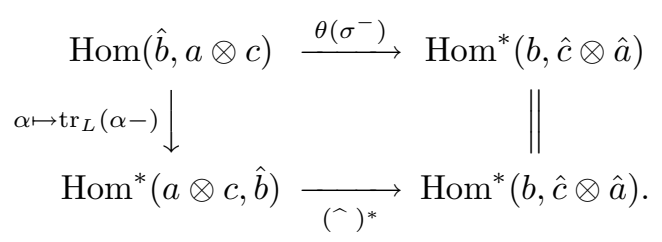

The map (^)* is the adjoint of the linear map ${ }^{\wedge}$.

From these diagrams, the action of the elements of $S^{4}$ on the linear functionals in the definition of the partition function can be computed. The maps $\phi$ and $\theta\left(\sigma^{+}\right)$ correspond, as diagrams, to rotations by one third of a turn, and ${ }^{\wedge}$ corresponds to one half of a turn. For even permutations in $S_{4}$, the symmetry property of the partition function follows from the fact that any even permutation of the vertices of a tetrahedron can be extended to an isotopy of the sphere. The definition of spherical category was constructed to give invariants of isotopy classes of graphs on the sphere. For odd elements of $S_{4}$, the symmetry property follows from the fact that any odd permutation of the vertices of a tetrahedron can be extended to an isotopy of the sphere which takes the tetrahedron to its image under some fixed reflection in a diameter. The diagrams corresponding to $A$ and $-A$ differ by such a reflection.

Alternatively, the symmetry property can be checked algebraically. As an example, consider the odd permutation $(0,1,2,3) \mapsto(3,1,2,0)$. The state space of $A$ is

$$
H\left(e_{23}, e_{13}, e_{12}\right) \otimes H^{*}\left(e_{23}, e_{03}, e_{02}\right) \otimes H\left(e_{13}, e_{03}, e_{01}\right) \otimes H^{*}\left(e_{12}, e_{02}, e_{01}\right)
$$

and the state space of $A^{\prime}$ is

$$
H^{*}\left(e_{23}^{\prime}, e_{13}^{\prime}, e_{12}^{\prime}\right) \otimes H\left(e_{23}^{\prime}, e_{03}^{\prime}, e_{02}^{\prime}\right) \otimes H^{*}\left(e_{13}^{\prime}, e_{03}^{\prime}, e_{01}^{\prime}\right) \otimes H\left(e_{12}^{\prime}, e_{02}^{\prime}, e_{01}^{\prime}\right) .
$$


The linear map of state spaces is

$$
x \otimes y \otimes z \otimes t \mapsto \theta^{*-1}\left(\sigma^{-1}\right) t \otimes \theta^{*-1}(\tau) y \otimes \theta(\tau) z \otimes \theta(\sigma) x,
$$

where $\sigma$ is the permutation $(0,1,2) \mapsto(1,2,0)$, and $\tau$ is the permutation $(0,1,2) \mapsto$ $(2,1,0)$. As a map of linear functionals, this is the adjoint of the map

$$
\alpha \otimes \beta \otimes \gamma \otimes \delta \mapsto \theta(\sigma) \delta \otimes \hat{\beta} \otimes \hat{\gamma} \otimes \widehat{\theta(\sigma) \hat{\alpha}} .
$$

The symmetry property is equivalent to the identity

$$
\operatorname{tr}_{L}(\hat{\gamma}(\theta(\sigma) \delta \otimes 1)(1 \otimes \widehat{\theta(\sigma) \hat{\alpha}}) \hat{\beta})=\operatorname{tr}_{L}(\beta(1 \otimes \delta)(\alpha \otimes 1) \gamma),
$$

which holds in any spherical category.

\section{Piecewise-Linear manifolds}

The aim of this section is to describe a finite set of moves on the triangulations of a 3-manifold such that any two triangulations are related by a finite sequence of these moves. These moves are given by a theorem of Pachner. An extension of this result is possible to admit some singularities; the proof of this also yields an elementary reduction of Pachner's result in three dimensions to Alexander's result on stellar moves.

Let $\sigma^{n}$ be an $n$-simplex. For any $p$ and $q$, the complexes given by the joins $\partial \sigma^{p} * \sigma^{q}$ and $\sigma^{p} * \partial \sigma^{q}$ are triangulations of the solid ball, $B^{p+q}$, and have the same boundary, namely $\partial \sigma^{p} * \partial \sigma^{q}$.

Definition 4.1. For any $k$ such that $0 \leqslant k \leqslant n$, if $X$ is any $n$-manifold with an identification of a boundary component with $\partial \sigma^{k} * \partial \sigma^{n-k}$, then $X \cup \partial \sigma^{k} * \sigma^{n-k}$ is said to be obtained from $X \cup \sigma^{k} * \partial \sigma^{n-k}$ by a bistellar move of order $k$.

Example 4.2. Figure 7 shows a bistellar move of order 2 in a 3 -manifold.

On the left-hand side there are two tetrahedra with a common horizontal face. On the right there are three tetrahedra with a common vertical edge.

The bistellar move of order 3 is drawn in Figure 8.

Note that bistellar moves have the following properties:

1. The inverse of a bistellar move of order $k$ is a bistellar move of order $n-k$.

2. A bistellar move on a manifold with boundary does not change the triangulation of the boundary.

The main result which gives the application of the algebra to topology is proved in [15].

Theorem 4.3. Two closed piecewise-linear n-manifolds are equivalent if and only if they are related by a finite sequence of bistellar moves.

A slight extension of Pachner's result is possible, allowing closed 3-manifolds with singularities at vertices.

Definition 4.4. A singular manifold is a complex with simplexes of dimension at most three, such that the link of every edge is a circle and the link of every face is two points.

It follows from the conditions that the link of a vertex in a singular manifold is a surface. The additional condition for a singular manifold to be a closed manifold is that the link of every vertex is a 2 -sphere. 


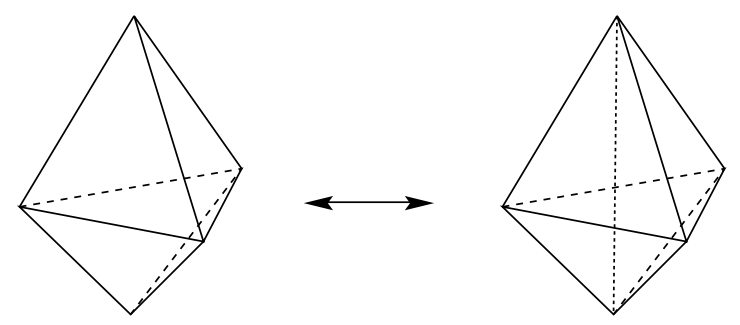

FiguRE 7
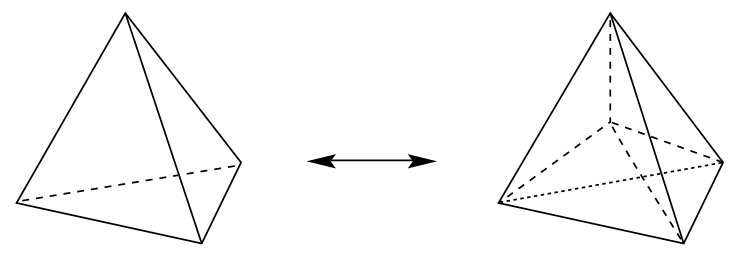

FiguRE 8

The result is based on the reduction of Alexander's stellar moves to bistellar moves. The theorem for stellar moves is given in $[8$, Chapter II, $\S \mathrm{D}$, Theorem II.17].

Theorem 4.5. Two finite simplicial complexes are piecewise-linear homeomorphic if and only if they are related by a finite sequence of stellar moves.

Our generalisation of Pachner's result is the following theorem.

Theorem 4.6. Two triangulated singular 3-manifolds are piecewise-linearly homeomorphic if and only if they are related by a finite sequence of bistellar moves.

Proof. It is sufficient to show that each stellar move can be obtained as a finite sequence of bistellar moves. In dimension three, this can be done explicitly. The stellar move on a 3 -simplex is a bistellar move. The stellar move on a triangle is a composite of two bistellar moves, using the condition that the link of the triangle is $S^{0}$. Finally, the stellar move on an edge is a composite of bistellar moves, using the condition that the link of an edge is a triangulation of $S^{1}$. This later construction is done explicitly as follows.

Let $N S$ be the vertices of an edge which is in $p$ tetrahedra. Label the vertices of these $p$ tetrahedra so that the vertices of these tetrahedra are

$$
N S E_{i} E_{i+1} \quad \text { for } 1 \leqslant i \leqslant p .
$$

This gives a triangulation of the 3-ball which looks like an orange with $p$ segments. Doing stellar subdivision on the edge $N S$ gives a triangulation of the 3 -ball with $2 p$ tetrahedra. This is like slicing the orange in half, cutting each segment in half.

In order to obtain this complex from the original one by bistellar moves, first do a bistellar move of order 4 on the tetrahedron $N S E_{1} E_{2}$. This introduces a new vertex which we label $O$. This gives the right number of vertices but only $p+3$ tetrahedra. Now for $j=2,3, \ldots, n$ do a bistellar move of order 3 on the two tetrahedra $O N S E_{j}$ and $N S E_{j} E_{j+1}$. This has the effect of introducing the edge $O E_{j+1}$ and replaces the two tetrahedra by the three tetrahedra $O N E_{j} E_{j+1}, O S E_{j} E_{j+1}$ and $O N S E_{j+1}$. 
This results in a triangulation of the 3-ball with $2 p+1$ tetrahedra. Finally do a bistellar move of order 2 on the 3 tetrahedra $O N S E_{n}, O N S E_{1}$ and $N S E_{1} E_{n}$, replacing them by the two tetrahedra $O N E_{1} E_{n}$ and $O S E_{1} E_{n}$.

\section{INVARIANTS OF MANIFOLDS}

The following is the main theorem in this paper.

Theorem 5.1. A finite semisimple spherical category of non-zero dimension determines an invariant of oriented singular 3-manifolds.

Since closed (3-)manifolds are examples of singular 3-manifolds, this determines an invariant of closed manifolds. Throughout this section, the proof refers to closed manifolds, which, as is the general convention in this paper, are taken to be oriented. However every statement is also true for oriented singular manifolds.

Let $M$ be a closed simplicial manifold, $J$ a choice of one simple object from each isomorphism class, and $K$ the dimension of the spherical category.

The notation in this section is as follows: for a simplicial manifold $M$, the edge set is denoted $E$. Thus $l: E \rightarrow I$ is a labelling, and the labelled manifold is the pair $(M, l)$. Let $v$ be the number of vertices of $M$.

Define the state sum invariant of $M$ by a summation over the set of all labellings by elements of $J$ :

$$
C(M)=K^{-v} \sum_{l: E \rightarrow J} Z(M, l) \prod_{e \in E} \operatorname{dim}_{q}(l(e)) .
$$

Proof of Theorem 5.1. The rest of this section is a proof that $C(M)$ is the manifold invariant; that is, that any simplicial manifold $M$ which triangulates a given piecewise-linear manifold $\mathcal{M}$ determines the same invariant.

Let $M$ be a simplicial complex that triangulates $\mathcal{M}$. First, $C(M)$ does not depend on the choice of simple objects $J$ due to Proposition 3.3. Next it is necessary to show that $C(M)$ does not depend on the choice of simplicial structure for the complex which triangulates $\mathcal{M}$, and finally that it does not depend on the choice of triangulation.

Let $M_{1}$ and $M_{2}$ be two different choices of simplicial structure with the same underlying complex and the same orientation. Then the identity map of complexes is a combinatorial isomorphism of simplicial manifolds. By Theorem 3.9, the state sum $C\left(M_{1}\right)$ is equal to a state sum over the set of labellings of $M_{2}$ which are compatible with a labelling $E \rightarrow J$ of $M_{1}$. This is not the state sum $C\left(M_{2}\right)$, because the labelling of an edge in the complex runs over either the set $J$ or the set $\hat{J}=\{\hat{a} \mid a \in J\}$. However it is equal to $C\left(M_{2}\right)$ because $\hat{a}$ is a simple object if $a$ is, and $\hat{J}$ also contains one element of each isomorphism class of simple objects. The equality follows from Proposition 3.3. This shows that $C(M)$ does not depend on the simplicial structure. Now it remains to consider the triangulation.

If $L$ is a subcomplex of a complex $M$, and $L$ has a simplicial structure determined by a total order of the vertices of $L$, then this can be extended to a simplicial structure of $M$, by extending the total order. If a complex $N$ is obtained from the complex $M$ by a bistellar move, so that $M=X \cup \sigma^{k} * \partial \sigma^{3-k}$ and $N=X \cup \partial \sigma^{k} * \sigma^{3-k}$, then a choice of standard simplicial structure for $\partial \sigma^{4}$ can be extended to $X \cup \partial \sigma^{4}$, which contains $M$ and $N$ as subcomplexes. Such a choice of simplicial structure for $\partial \sigma^{4}$ is just the identification of $\sigma^{4}$ as the boundary of the standard 4-simplex, (01234). 
Let $(i j) \mapsto e_{i j}$, for $0 \leq i<j \leq 4$ be a labelling of the standard 4-simplex (01234). This determines partition functions for each tetrahedron in the boundary,

$$
\begin{gathered}
Z( \pm(1234))=\left\{\begin{array}{lll}
e_{12} & e_{13} & e_{23} \\
e_{34} & e_{24} & e_{14}
\end{array}\right\}_{ \pm}, \quad Z( \pm(0234))=\left\{\begin{array}{lll}
e_{02} & e_{03} & e_{23} \\
e_{34} & e_{24} & e_{04}
\end{array}\right\}_{ \pm} \\
Z( \pm(0134))=\left\{\begin{array}{lll}
e_{01} & e_{03} & e_{13} \\
e_{34} & e_{14} & e_{04}
\end{array}\right\}_{ \pm}, \quad Z( \pm(0124))=\left\{\begin{array}{lll}
e_{01} & e_{02} & e_{12} \\
e_{24} & e_{14} & e_{04}
\end{array}\right\}_{ \pm} \\
Z( \pm(0123))=\left\{\begin{array}{lll}
e_{01} & e_{02} & e_{12} \\
e_{23} & e_{13} & e_{03}
\end{array}\right\}_{ \pm}
\end{gathered}
$$

The invariance of $C(M)$ under bistellar moves follows from the next proposition.

Let $P$ be the map $x \otimes y \mapsto y \otimes x$.

Proposition 5.2 (Orthogonality). The map

$$
\operatorname{dim}_{q}\left(e_{02}\right) \sum_{e_{13} \in J} Z(0123) Z(-0123) \operatorname{dim}_{q}\left(e_{13}\right)
$$

is equal to the identity map on $H\left(e_{23}, e_{03}, e_{02}\right) \otimes H\left(e_{12}, e_{02}, e_{01}\right)$.

(Biedenharn-Elliot). The equality

$$
\begin{aligned}
& (Z(0234) \otimes 1)(1 \otimes Z(0124)) \\
& \quad=\sum_{e_{13} \in J} \operatorname{dim}_{q}\left(e_{13}\right)(1 \otimes Z(0123))(P \otimes 1)(1 \otimes Z(0134))(P \otimes 1)(Z(1234) \otimes 1)
\end{aligned}
$$

holds.

These equalities hold for all choices of labels $\left\{e_{i j}\right\}$ not explicitly summed over.

The proof of these will be given below, after completing the proof of Theorem 5.1. Theorem 5.1 will follow once it has been established that $C(M)$ is invariant under bistellar moves of orders 2 and 3. The bistellar moves of order 1 and 0 are the inverses of these moves.

The simplicial invariant can be decomposed as $Z\left(M, l_{M}\right)=\operatorname{tr}\left(Z(X), Z\left(D^{1}\right)\right)$ and $Z\left(N, l_{N}\right)=\operatorname{tr}\left(Z(X), Z\left(D^{2}\right)\right)$, where $D^{1}$ and $D^{2}$ are the simplicial disks in the bistellar moves, $D^{1} \cup D^{2}=\partial(01234), M=X \cup D^{1}, N=X \cup D^{2}$, and $X \cup(01234)$ is labelled with restriction $l_{M}$ to $M$ and $l_{N}$ to $N$. The linear map $Z(X)$ is defined to be the partial trace over the state spaces of all triangles not in the boundary of $X$ of the tensor product of the partition functions for each oriented labelled tetrahedron in $X$. The linear maps $Z\left(D^{1}\right)$ and $Z\left(D^{2}\right)$ are defined likewise.

The invariance of $C(M)$ under bistellar moves follows by establishing that

$$
K^{-v^{1}} \sum_{l}\left(Z\left(D^{1}\right) \prod_{e}\left(\operatorname{dim}_{q}(l(e))\right)\right)=K^{-v^{2}} \sum_{l}\left(Z\left(D^{2}\right) \prod_{e}\left(\operatorname{dim}_{q}(l(e))\right)\right) .
$$

In this formula, $v^{1}, v^{2}$ are the number of vertices internal to $D^{1}, D^{2}$ (i.e., not on the boundary); the product is over edges internal to $D^{1}$ or $D^{2}$, and the summation is over labellings which are fixed on $\partial D^{1}=\partial D^{2}$ but range over all values in $J$ for all edges internal to $D^{1}$ or $D^{2}$.

For the bistellar move of order 2 , there are no internal vertices and the equality is the Biedenharn-Elliot identity of Proposition 5.2.

For the bistellar move of order 3 , the required identity is 


\section{Lemma 5.3.}

$$
\begin{array}{r}
Z(0234)=K^{-1} \sum_{\substack{e_{01}, e_{12}, e_{13}, e_{14} \in J}}\left(\operatorname{tr}_{3}((1 \otimes Z(0123))(P \otimes 1)(1 \otimes Z(0134))(P \otimes 1)\right. \\
\left.\cdot(Z(1234) \otimes 1)(1 \otimes Z(-0124))) \prod_{n=0,2,3,4} \operatorname{dim}_{q}\left(e_{1 n}\right)\right),
\end{array}
$$

in which $\operatorname{tr}_{3}$ is the partial trace over the third factor:

$$
(\alpha \otimes \beta \otimes \gamma) \mapsto \alpha \otimes \beta \operatorname{tr}(\gamma) .
$$

Proof. Follow the linear maps on each side of the Biedenharn-Elliot relation with the linear map

$$
K^{-1} \operatorname{dim}_{q}\left(e_{01}\right) \operatorname{dim}_{q}\left(e_{12}\right) \operatorname{dim}_{q}\left(e_{14}\right)(1 \otimes Z(-0124)),
$$

take the partial trace on the third factor and sum over $e_{01} \in J, e_{12} \in J$, and $e_{14} \in J$. The right-hand side of the Biedenharn-Elliot identity becomes the right-hand side of the equation in the statement of the lemma. The left-hand side becomes

$$
\begin{array}{r}
K^{-1} \sum_{e_{01}, e_{12}, e_{14} \in J} \operatorname{tr}_{3}((Z(0234) \otimes 1)(1 \otimes Z(0124))(1 \otimes Z(-0124))) \operatorname{dim}_{q}\left(e_{01}\right) \\
\cdot \operatorname{dim}_{q}\left(e_{12}\right) \operatorname{dim}_{q}\left(e_{14}\right) .
\end{array}
$$

Using the orthogonality relation of Proposition 5.2, this is equal to

$$
\frac{K^{-1}}{\operatorname{dim}_{q}\left(e_{02}\right)} \sum_{e_{01}, e_{12} \in J} \operatorname{tr}_{3}((Z(0234) \otimes 1)) \operatorname{dim}_{q}\left(e_{01}\right) \operatorname{dim}_{q}\left(e_{12}\right) \text {. }
$$

Also,

$$
\operatorname{tr}_{3}((Z(0234) \otimes 1))=Z(0234) \operatorname{dim} \operatorname{Hom}\left(e_{02}, e_{12} \otimes e_{01}\right),
$$

using the ordinary vector space dimension dim. From the symmetry conditions and Lemma 2.14, it follows that

$$
\begin{aligned}
\sum_{e_{01}} \operatorname{dim}_{q}\left(e_{01}\right) \operatorname{dim} \operatorname{Hom}\left(e_{02}, e_{12} \otimes e_{01}\right) & =\sum_{e_{01}} \operatorname{dim}_{q}\left(e_{01}\right) \operatorname{dim} \operatorname{Hom}\left(e_{01}, \hat{e}_{12} \otimes e_{02}\right) \\
& =\operatorname{dim}_{q}\left(e_{12}\right) \operatorname{dim}_{q}\left(e_{02}\right) .
\end{aligned}
$$

Thus the left-hand side of the relation is equal to

$$
K^{-1} Z(0234) \sum_{e_{12} \in J}\left(\operatorname{dim}_{q}\left(e_{12}\right)\right)^{2}=Z(0234) .
$$

Thus the lemma is proved.

To complete the proof of Theorem 5.1, it remains to prove Proposition 5.2. The first step is

Lemma 5.4 (Crossing). The following diagram is commutative:

$$
\begin{array}{ccc}
\bigoplus_{e_{02} \in J} H\left(e_{23}, e_{03}, e_{02}\right) \otimes H\left(e_{12}, e_{02}, e_{01}\right) & \stackrel{\Phi}{\longrightarrow} \bigoplus_{e_{13} \in J} H\left(e_{23}, e_{13}, e_{12}\right) \otimes H\left(e_{13}, e_{03}, e_{01}\right) \\
& & \alpha \otimes \beta \mapsto \beta(\alpha \otimes 1) \downarrow \\
\operatorname{Hom}\left(e_{03}, e_{23} \otimes\left(e_{12} \otimes e_{01}\right)\right) & = & \operatorname{Hom}\left(e_{03},\left(e_{23} \otimes e_{12}\right) \otimes e_{01}\right)
\end{array}
$$


where the linear map

$$
\Phi=\bigoplus_{\substack{e_{2} \in J \\
e_{13} \in J}} \operatorname{dim}_{q}\left(e_{13}\right)\left\{\begin{array}{lll}
e_{01} & e_{02} & e_{12} \\
e_{23} & e_{13} & e_{03}
\end{array}\right\}_{+} .
$$

Also, the linear map

$$
\Psi=\bigoplus_{\substack{e_{02} \in J \\
e_{13} \in J}} \operatorname{dim}_{q}\left(e_{02}\right)\left\{\begin{array}{lll}
e_{01} & e_{02} & e_{12} \\
e_{23} & e_{13} & e_{03}
\end{array}\right\} .
$$

is the inverse of $\Phi$.

Proof. The semisimple condition implies that the vertical arrows are isomorphisms. Consider an element $\alpha \otimes \beta$ in the top left-hand space. Taking the trace of the two images of this element in the bottom right-hand space with $(\gamma \otimes 1) \delta$ for arbitrary $\gamma \in \operatorname{Hom}\left(e_{23} \otimes e_{12}, e_{13}\right)$ and $\delta \in \operatorname{Hom}\left(e_{13} \otimes e_{01}, e_{03}\right)$ yields two elements of $\mathbb{F}$ which are equal. This shows that the diagram commutes. Replacing the map $\Phi$ with the inverse of $\Psi$ also yields a commutative diagram, by a similar argument. Combining these two diagrams shows that $\Phi=\Psi^{-1}$.

Proof of Proposition 5.2. The proof of the orthogonality relation is a direct consequence of Lemma 5.4. The Biedenharn-Elliot identity follows from the fact that the following diagram commutes (in this diagram the shorthand notation (012) is used for the state space of this triangle, $\left.\operatorname{Hom}\left(e_{02}, e_{12} \otimes e_{01}\right)\right)$ :

$$
\begin{gathered}
\bigoplus_{e_{02}, e_{03} \in J}(034) \otimes(023) \otimes(012) \stackrel{1 \otimes Z(0123)}{\longrightarrow} \bigoplus_{e_{03}, e_{13} \in J}(034) \otimes(123) \otimes(013) \\
Z(0234) \otimes 1 \downarrow \\
\bigoplus_{e_{13}, e_{14} \in J}(123) \otimes(134) \otimes(014) \\
\bigoplus_{e_{02}, e_{24} \in J}(234) \otimes(024) \otimes(012) \stackrel{1 \otimes Z(0124)}{\longrightarrow} \bigoplus_{e_{14}, e_{24} \in J}(234) \otimes(124) \otimes(014)
\end{gathered}
$$

Applying Lemma 5.4 five times shows that this diagram commutes.

Remarks. The idea of using a state sum model to construct manifold invariants is due to [25], where an invariant is constructed from the $6 j$-symbols of $U_{q} \mathfrak{s l}_{2}$, for $q$ a root of unity. Every state space $H(a, b, c)$ is 0 or $\mathbb{C}$ and every map $\theta(\sigma): V \sigma(a, b, c) \rightarrow V(a, b, c)$ is the identity. Hence the partition function of a labelled tetrahedron is simply a number, and these numbers are equal under permutations of the labels. These numbers are the quantum analogues of the $6 j$-symbols. The identities of Proposition 5.2 are the quantum analogues of the well-known Biedenharn-Elliot and orthogonality identities, as proved in [18].

This example satisifies some extra conditions, which entail firstly that the invariant is defined for unoriented manifolds, and secondly that there is a topological quantum field theory associated to the invariant.

The first condition is that each space $H(a, b, c)$ is an inner product space and that the partition function of $-A$ is the adjoint of the partition function of $A$ with 
respect to these inner products. This condition implies that the invariant is defined for unoriented manifolds.

The second condition is that each self-dual simple object in the category is orthogonal and not symplectic. If $a$ is a self-dual simple object, then there is an isomorphism $\phi: a \rightarrow \hat{a}$. This object is called orthogonal if $\phi=\hat{\phi}$, or symplectic if $\phi=-\hat{\phi}$. These are the only possibilities, as ${ }^{-}$is an involution. This classification does not depend on the choice of isomorphism $\phi$, as $\operatorname{Hom}(a, \hat{a}) \cong \mathbb{F}$ and ${ }^{\wedge}$ is linear.

This condition is necessary for the construction of a topological field theory by a construction similar to that of [25], because it is necessary for the isomorphisms of naturality (Definition 3.1) and symmetry (Definition 3.7) on the state space of a triangle to commute. The condition is a sufficient condition because it is a coherence condition which allows the construction of a strict spherical category in which $\hat{a}=a$ for all self-dual objects.

For the example of $U_{q} \mathfrak{s l}_{2}$, there are exactly two choices of the element $w$ which make this Hopf algebra spherical. The topological field theory of [25] is constructed with the element for which every simple module is orthogonal. At the value $q=1$, this element $w$ takes the value 1 on the even (integer spin) representations and -1 on the odd (odd half-integer spin) representations.

\section{Spherical Hopf algebras}

Definition 6.1. A spherical Hopf algebra over a field $k$ consists of a finite dimensional vector space $A$ together with the following data:

1. a multiplication $\mu$,

2. a unit $\eta: \mathbb{F} \rightarrow A$,

3. a comultiplication $\Delta: A \rightarrow A \otimes A$,

4. a counit $\epsilon: A \rightarrow \mathbb{F}$,

5. an antipodal map $\gamma: A \rightarrow A$,

6. an element $w \in A$.

The data $(A, \mu, \eta, \Delta, \epsilon, \gamma)$ is required to define a Hopf algebra. The conditions on the element $w$ are the following:

1. $\gamma^{2}(a)=w a w^{-1}$ for all $a \in A$.

2. $\Delta(w)=w \otimes w$.

3. $\operatorname{tr}(\theta w)=\operatorname{tr}\left(\theta w^{-1}\right)$ for all left $A$-modules $V$ and all $\theta \in \operatorname{End}_{A}(V)$.

It follows from the condition $\Delta(w)=w \otimes w$ that $\gamma(w)=w^{-1}$ and that $\epsilon(w)=1$. Such elements are called group-like.

Example 6.2. Examples of Hopf algebras which are spherical are:

1. Any involutory Hopf algebra is spherical. The element $w$ can be taken to be 1.

2. Any ribbon Hopf algebra, as defined in [19], is spherical. The element $w$ can be taken to be $u v^{-1}$, where the element $u$ is determined by the quasi-triangular structure and the element $v$ is the ribbon element.

Remark 6.3. A Hopf algebra with an element $w$ that satisfies the first two conditions of Definition 6.1 is spherical if either $w^{2}=1$, or all modules are isomorphic to their dual. 
Remark 6.4. If $A$ is a Hopf algebra, there may exist more than one element $w$ such that $(A, w)$ is a spherical Hopf algebra. However, if $w_{1}$ is one such element, then $w_{2}=g w_{1}$ is another such element if and only if $g$ satisfies the conditions:

1. $g$ is central,

2. $g$ is group-like,

3. $g$ is an involution.

Example 6.5. This is an example of a finite dimensional Hopf algebra which satisfies all the conditions for a spherical Hopf algebra except that the left and right traces are distinct. This example is the quantised enveloping algebra of the Borel subalgebra of $\mathfrak{s l}_{2}(\mathbb{C})$.

Let $s$ be a primitive $2 r$-th root of unity with $r>1$. Let $B$ be the unital algebra generated by elements $X$ and $K$ subject to the defining relations

$$
\begin{aligned}
K X & =s X K, \\
K^{4 r} & =1, \\
X^{r} & =0 .
\end{aligned}
$$

Then $B$ is a finite dimensional algebra and also has a Hopf algebra structure defined by:

1. The coproduct, $\Delta$, is defined by $\Delta(K)=K \otimes K$ and $\Delta(X)=X \otimes K+K^{-1} \otimes X$,

2. The augmentation, $\epsilon$, is defined by $\epsilon(K)=1$ and $\epsilon(X)=0$,

3. The antipode, $\gamma$, is defined by $\gamma(K)=K^{-1}$ and $\gamma(X)=-s X$.

The element $w=K^{2}$ satisfies the conditions

$$
\begin{aligned}
\Delta(w) & =w \otimes w, \\
\epsilon(w) & =1, \\
\gamma(w) & =w^{-1}, \\
\gamma^{2}(b) & =w b w^{-1} \quad \text { for all } b \in B .
\end{aligned}
$$

The trace condition is not satisfied since $B$ has $4 r$ one dimensional representations with $X=0$ and $K$ a $4 r$-th root of unity, and it is clear that the trace condition is not satisfied in these representations.

Example 6.6. Not all spherical Hopf algebras are modular Hopf algebras in the sense of [17]. For example, the group algebra of a finite group over a field of characteristic 0 , or more generally, any cocommutative, involutory, semisimple Hopf algebra, is spherical and not modular.

Also, not all spherical Hopf algebras are ribbon Hopf algebras. The dual of the group algebra of a non-commutative finite group can be made a spherical Hopf algebra. However, the representation ring is non-commutative, and so the Hopf algebra cannot be quasi-triangular.

The next proposition gives the application of spherical Hopf algebras to state sum models.

Proposition 6.7. The category of representations of a spherical Hopf algebra, A, is equivalent to a canonical strict spherical category.

In this category, the objects are lists of left $A$-modules and the morphisms $A$ linear maps of the modules formed by tensor product over the list. The trace 
$\operatorname{tr}_{L}$ of an endomorphism $\theta$ of a left $A$-module is the matrix trace of $\theta w$. The full construction is given in [3].

Some extra conditions are required to give invariants of manifolds. The first way this can be achieved uses the same data as the construction of invariants given in [11].

Proposition 6.8. Let $A$ be a finite dimensional involutory Hopf algebra over an algebraically closed field of characteristic 0. Then A determines a 3-manifold invariant.

Proof. The Hopf algebra $A$ gives a spherical Hopf algebra by taking $w=1$. In this case the quantum trace is just the matrix trace. The algebra $A$ is semisimple by [12]. Hence the category of finite dimensional left $A$-modules is a spherical category which satisfies the hypotheses of Theorem 5.1.

In general, the construction of the non-degenerate quotient is another way of attaining the semisimple condition. The following proposition is proved in [3].

Proposition 6.9. Let $A$ be a spherical Hopf algebra over an algebraically closed field. Then the non-degenerate quotient of the spherical category of finitely generated left A-modules is semisimple.

A corollary to the proof of this proposition is that the nondegenerate quotient of any spherical subcategory of the category of left $A$-modules which is closed under taking direct summands is a semisimple spherical category. Thus, in order to construct a manifold invariant, it is sufficient to construct such spherical subcategories which are finite and have non-zero dimension.

Let $A$ be a finite dimensional spherical Hopf algebra. If $A$ is semisimple as an algebra, then it is clear that the non-degenerate quotient of the category of left $A$ modules satisfies all the hypotheses of Theorem 5.1 except possibly the condition that $K=0$. In the following discussion we assume that $A$ is not semisimple. In this case the set of isomorphism classes of simple objects in the non-degenerate quotient may well be infinite. This may come about since each $A$-module $V$ that satisfies $\operatorname{End}_{A}(V) \cong \mathbb{F}$ and which has non-zero quantum dimension gives a simple object in the quotient. The condition that $\operatorname{End}_{A}(V) \cong \mathbb{F}$ is much weaker than the condition that $V$ is irreducible. Although it is possible for inequivalent modules to give equivalent simple objects in the quotient, there is no apparent reason for the set of simple objects in the quotient to be finite, in general.

Hence in order to construct a manifold invariant from a spherical Hopf algebra $A$ which is not semisimple it is necessary to find a proper spherical subcategory of the category of left $A$-modules such that the non-degenerate quotient is finite and has non-zero dimension. A subcategory of a spherical category is a spherical subcategory if and only if it is closed under addition, tensor product and taking duals.

The category of left $A$-modules has a proper spherical subcategory, namely the category of projective left $A$-modules. This category is spherical, since it is closed under tensor product and is closed under taking duals by [13]. However, the next proposition is a negative result which shows that this spherical subcategory cannot be used to construct manifold invariants.

Proposition 6.10. Let $A$ be a finite dimensional pivotal Hopf algebra which is not semisimple. Then every projective A-module has zero quantum dimension. 
Proof. If $A$ is any Hopf algebra, then the linear functional

$$
a \mapsto \operatorname{tr}\left(x \mapsto \gamma^{-2}(x a)\right)
$$

is a right co-integral. If $A$ is not a semisimple algebra this is identically zero [12, Proposition 2.4]; and so if $A$ satisfies the hypotheses of the proposition then, for all $a \in A, \operatorname{tr}\left(x \mapsto w^{-1} x a w\right)=0$. Now choose a primitive idempotent $\pi$ and put $a=\pi \omega^{-1}$. This gives

$$
0=\operatorname{tr}\left(x \mapsto w^{-1} x \pi\right)=\operatorname{dim}_{q}(A \pi),
$$

which shows that every indecomposable projective $A$-module has zero quantum dimension.

Examples of spherical Hopf algebras which are not semisimple are quantised enveloping algebras at roots of unity. The following theorem shows that each of these examples gives a manifold invariant. This theorem is proved in [1] for odd roots of unity and in [2] in general. The main steps of the argument follow.

Theorem 6.11. Let $A$ be a quantised enveloping algebra of a finite dimensional semisimple Lie algebra at a root of unity. Assume that the order, $k$, of the quantum parameter $q$ is at least the Coxeter number of the Lie algebra. Then the category of tilting modules is spherical and the non-degenerate quotient is finite with non-zero dimension, satisfying the hypotheses of Theorem 5.1.

Proof. It follows from the definition that the category of tilting modules is closed under taking duals and direct summands. The set of tilting modules is also closed under tensor product. The isomorphism classes of indecomposable tilting modules are indexed by the dominant weights, and the quantum dimension is zero unless the dominant weight lies in the interior of the fundamental alcove. In particular this shows that the set of isomorphism classes of simple objects in the non-degenerate quotient is finite. Each tilting module corresponding to a dominant weight in the interior of the fundamental alcove is a Weyl module (as well as being irreducible), and so the quantum dimension is given by the Kac formula. The quantum dimension of the dual is given by substituting $q^{-1}$ for $q$ in this formula. Since $\operatorname{dim}_{q}(V)=$ $\operatorname{dim}_{q}\left(V^{*}\right)$ for any $V$ and $q$ has unit modulus, it follows that each quantum dimension is real. Hence the dimension $K$ is a sum of positive real numbers. The condition that $k$ is greater than or equal to the Coxeter number ensures that there is at least one dominant weight in the interior of the fundamental alcove, and so $K$ is non-zero.

\section{REFERENCES}

[1] H. H. Andersen, Tensor products of quantized tilting modules, Comm. Math. Phys. 149 (1992), 149-159. MR 94b:17015

[2] H. H. Andersen and J. Paradowski, Fusion categories arising from semisimple Lie algebras, Comm. Math. Phys. 169 (1995), 563-588. MR 96e:17026

[3] J. W. Barrett and B. W. Westbury, Spherical categories, preprint, hep-th/9310164, University of Nottingham, 1993.

[4] J. W. Barrett and B. W. Westbury, The equality of 3-manifold invariants, Math. Proc. Cambridge Philos. Soc. 118 (1995), 503-510.

[5] B. Durhuus, H. P. Jakobsen and R. Nest, Topological quantum field theories from generalized 6j-symbols, Reviews in Math. Physics 5 (1993), 1-67. MR 94h:57025

[6] P. J. Freyd and D. N. Yetter, Braided compact closed categories with applications to low dimensional topology, Adv. in Math. 77 (1989), 156-182. MR 91c:57019 
[7] P. Freyd and D. N. Yetter, Coherence theorems via knot theory, J. Pure Appl. Algebra 78 (1992), 49-76. MR 93d:18013

[8] L. C. Glaser, Geometrical Combinatorial Topology I, Van Nostrand Reinhold Mathematical Studies 27 (1970).

[9] A. Joyal and R. Street, The geometry of tensor calculus, I, Adv. in Math. 88 (1991), 55-112. MR 92d:18011

[10] G. M. Kelly and M. I. Laplaza, Coherence for compact closed categories, J. Pure Appl. Algebra 19 (1980), 193-213. MR 81m:18008

[11] G. Kuperberg, Involutory Hopf algebras and 3-manifold invariants, International Journal of Mathematics 2 (1991), 41-66. MR 91m:57012

[12] R. G. Larson and D. E. Radford, Finite dimensional cosemisimple Hopf algebras in characteristic 0 are semisimple, J. Algebra (1988), 267-289. MR 89k:16016

[13] R. G. Larson and M. E. Sweedler, An associative orthogonal bilinear form for Hopf algebras, Amer. J. Math. 91 (1969), 75-94. MR 39:1523

[14] J. P. Moussouris, Quantum models of space-time based on coupling theory, D. Phil. Oxford (1983).

[15] U. Pachner, P.l. homeomorphic manifolds are equivalent by elementary shellings, European Journal of Combinatorics 12 (1991), 129-145. MR 92d:52040

[16] G. Ponzano and T. Regge, Semiclassical limit of Racah coefficients, in Spectroscopic and Group Theoretical Methods in Physics, North-Holland, Amsterdam, 1968, pp. 1-58.

[17] N. Reshetikhin and V. G. Turaev, Invariants of 3-manifolds via link polynomials and quantum groups, Invent. Math. 103 (1991), 547-597. MR 92b:57024

[18] N. Y. Reshetikhin and A. N. Kirillov, Representations of the algebra $U_{q}(\operatorname{sl}(2))$, q-orthogonal polynomials and invariants of links in Infinite-dimensional Lie Algebras and Groups, (V. G. Kac, ed.), World Scientific, Singapore, 1988, pp. 285-339. MR 90m:17022

[19] N. Y. Reshetikhin and V. G. Turaev, Ribbon graphs and their invariants derived from quantum groups, Comm. Math. Phys. 127 (1990), 1-26. MR 91c:57016

[20] J. Roberts, Skein theory and Turaev-Viro invariants, Topology 34 (1995), 771-787.

[21] C. P. Rourke and B. J. Sanderson, Introduction to piecewise-linear topology, Springer-Verlag, New York-Heidelberg-Berlin, 1982. MR 83g:57009

[22] V. Turaev, Quantum invariants of 3-manifold and a glimpse of shadow topology, in Quantum Groups (Leningrad, 1980), Lecture Notes in Math. 1510, Springer-Verlag, Berlin, 1992, pp. 363-366. MR 93j:57010

[23] V. G. Turaev, Modular categories and 3-manifold invariants, Internat. J. Modern Phys. 6 (1992), 1807-1824. MR 93k:57040

[24] V. G. Turaev, Quantum Invariants of Knots and 3-manifolds, De Gruyter, New York-Berlin, 1994. MR 95k:57014

[25] V. G. Turaev and O. Y. Viro, State sum invariants of 3-manifolds and quantum $6 j$-symbols, Topology 31 (1992), 865-902. MR 94d:57044

[26] V. Turaev and H. Wenzl, Quantum invariants of 3-manifolds associated with classical simple Lie algebras, International Journal of Mathematics 4 (1993), 323-358. MR 94i:57019

[27] K. Walker, On Witten's 3-manifold invariants, 1990.

[28] D. N. Yetter, State-sum invariants of 3-manifolds associated to Artinian semisimple tortile categories, Topology and its Applications 58 (1993), 47-80. MR 95g:57032

Department of Mathematics, University of Nottingham, University Park, NottingHAM, NG7 2RD, U.K.

E-mail address: jwb@maths.nott.ac.uk

E-mail address: bww@maths.nott.ac.uk 